\title{
Virtual biopsies and vibrational-mechanical analysis of skin rejuvenation: comparison of results obtained with micro-needling and topical treatments
}

\begin{abstract}
Vibrational optical coherence tomography (VOCT) is a new non-invasive and nondestructive technique that can be used to image and measure the mechanical properties of skin. The technique uses infrared light and audible sound to image and measure the resonant frequency of tissue components. The stiffness (modulus) of each major tissue component is obtained from a calibration equation using values of the measured resonant frequency and tissue thickness. VOCT is used in this study to quantitatively compare the outcomes of cell proliferation and fibrous collagen deposition as a result of (1) a skin topical treatment (CE Ferulic) and (2) micro-needle roller application to human forehead skin. Both methods appear to quantitatively increase the ratio of cells to dermal collagen and the ratio of fibrotic collagen to dermal collagen with increasing time. Both chemical and mechanical treatments lead to cell proliferation and new collagen deposition using these skin rejuvenation procedures. While results obtained with micro-needle rolling are achieved more rapidly, collagen deposition with the topical is achieved painlessly. The new collagen that is deposited, using both methods, appears similar to that seen in wound healing and has a higher resonant frequency and modulus compared to normal dermal collagen. The results of this study suggest that VOCT can provide useful quantitative data to evaluate the efficacy of skin rejuvenation procedures. It is suggested that there is a need to develop techniques to regenerate normal dermal collagen as opposed to fibrotic collagen to reduce the stiffness of the new tissue formed.
\end{abstract}

Keywords: micro-needling, CE Ferulic, topical treatment, skin, epidermis, keratinocytes, epidermal cells, virtual biopsy, dermal collagen, fibrotic collagen
Volume 4 Issue 3 - 2020

\author{
Frederick H Silver, ${ }^{1,2}$ Nikita Kelkar ${ }^{2}$ \\ 'Department of Pathology and Lab Medicine, Robert Wood \\ Johnson Medical School, Rutgers, the State University of New \\ Jersey, USA \\ ${ }^{2}$ OptoVibronex, LLC., USA
}

Correspondence: Frederick H Silver, Department of Pathology and Laboratory Medicine, Robert Wood Johnson Medical School, Rutgers, the State University of New Jersey, USA, Email fhsilver@hotmail.com

Received: May 19, 2020 | Published: May 25, 2020
Abbreviations: OCT, optical coherence tomography; VOCT, vibrational optical coherence tomography

\section{Introduction}

Intrinsic and environmental extrinsic factors lead to skin aging in humans that produces an undesirable look that includes wrinkling. ${ }^{1}$ Methods developed to rejuvenate a youthful skin look include use of skin fillers such as hyaluronic acid, ${ }^{1,2}$ introduction of topical drugs such as Retinoids, ${ }^{3}$ delivery of substances to cutaneous and subcutaneous tissues through micro-needling and topical patches, ${ }^{4}$ and the use of cosmeceuticals such as ascorbic and glycolic acids that penetrate into the skin. ${ }^{5}$

Skin is a multilayered tissue consisting of cells, macromolecules, and water, separated into several distinct layers. ${ }^{6,7}$ The epidermis, found at the air-skin interface, is between $0.7 \mathrm{~mm}$ and $1.2 \mathrm{~mm}$ in thickness. ${ }^{6,7}$ Below the epidermis is the papillary and reticular dermal layers which are between 1 and $4 \mu \mathrm{m}$ thick. ${ }^{7}$ The papillary layer occupies about $10 \%$ of the dermal thickness and contains type I and a small amount of type III collagen in fibrils 20 to $40 \mu \mathrm{m}$ in diameter. ${ }^{7}$ The fibrils are packed into collagen fibers 0.3 to $3.0 \mathrm{~mm}$ in diameter. ${ }^{6}$ The reticular dermis is found below the papillary dermis and contains type I collagen fibrils $60-100 \mu \mathrm{m}$ in diameter. ${ }^{6}$ The mean collagen volume fractions for both papillary and reticular dermis are reported to between $66 \%$ and $69 \%$. Collagen fiber compaction and unraveling is observed to occur with increased age ${ }^{8,9}$ and contributes to skin wrinkling.

Elastic tissue is the other major macromolecular component of skin. The volume fraction of elastic tissue increases from about $0.7 \%$ to $2.5 \%$ and the fiber diameters increase from 1 to $2 \mu \mathrm{m}$ as one goes from the papillary to the recticular dermis. ${ }^{8,10}$ Elastic fibers from older individuals show fraying and hole formation ${ }^{8}$ that also contribute to skin wrinkling.

The presence of a barrier between the stratum corneum and viable epidermis was shown to exist ${ }^{11}$ as early as 1920 . The stratum corneum consists of keratinized cells 18 to 20 layers thick that are cemented together by lipids including ceramides that form intracellular lamellae. These intracellular lamellae form the basis of the permeability barrier and contain about 13 different types of lipids. ${ }^{11}$ Lipid enriched lamellar bodies, which compose $20 \%$ of the cell volume, are found at the interface between the stratum corneum and the stratum granulosum. These lamellar bodies form the permeability boundaries that limit difffusion of hydrophilic substances through the epidermis. ${ }^{11}$

A variety of methods have been used to promote release of drugs and diffusion of molecules through the stratum corneum-stratum granulosum barrier. They include transdermal patches, chemical enhancers, iontophoresis, sonophoresis, jet systems, radiofrequency ablation, laser ablation, ultrasound stimulation, biochemical enhancers, electroporation, micro-needling, thermal ablation and microdermabrasion..$^{12-16}$ The purpose of this paper is to introduce a new method, vibrational optical coherence tomography(VOCT) and the "virtual biopsy", as a tool to evaluate the use and efficacy of skin rejunvenation treatments. In this paper we compare the outcomes using a topical treatment, CE Ferulic, to that obtained using microneedling in the aesthestic rejunvention of forehead facial skin. 


\section{Methods}

\section{Patient consent}

Skin from ten subjects was studied in vivo after informed consent was obtained. The subjects ranged in age from 25 to 75 years of age with a mean age of 57 years old and no feeling of the light or sound reflecting from the skin was experienced by the subjects. Tissues examined include skin from the cheek, back of the hand, forehead, palm and skin above the radial artery in the wrist. A topical preparation CE Ferulic (15\% L-Ascorbic Acid, 1\% Alpha Tocopherol, 0.5\% Ferulic Acid, Skin Ceuticals, Dallas Tx) was applied daily to one of the subjects for a period of about 7.0 months and a micro-needle roller with a needle length of $1.5 \mathrm{~mm}$ (Micro540 Needles Derma Roller, HIEGOO, UK) was applied ten times to the forehead, after a $5 \%$ lidocaine cream, was applied on the forehead of a second subject at the beginning of the study. The skin was evaluated for a period of one month post-treatment. Images and resonant frequency changes of the site of topical drug application were evaluated for changes in cell and collagen contents using VOCT at periods of up to 7 months. Needling results were evaluated immediately after treatment and at 1,2 and 4 weeks post-treatment.

\section{Optical coherence tomography (OCT) image collection}

OCT image collection was accomplished using a VOCT device (Vibrational Opto Scope, OptoVibronex, LLC) in the scanning mode. All gray scale images were color coded to enhance the image details. The original gray scale OCT images of skin were pseudo color-coded based on the pixel intensities as described previously. ${ }^{17,18}$ The enhanced OCT images used darker colored (blue and purple) regions to reflect lower pixel intensities while the lighter (yellowish) regions reflected higher pixel intensity regions. Pixel intensities were processed using image J software, analyzed with a MATLAB program, and plotted versus skin depth.

\section{Vibrational optical coherence tomography (VOCT) and vibrational analysis in Vivo}

VOCT is a non-invasive and non-destructive method that uses infrared light and audible sound to create a displacement of skin as described in detail previously. ${ }^{17-19}$ The measured resonant frequencies are converted into modulus values using a calibration equation (equation 1) developed based on in vitro uniaxial mechanical tensile testing and VOCT measurements..$^{20,21}$

Experimentally, the VOCT hand piece uses infrared light to image the specimen as described previously. ${ }^{17-21}$ The infrared beam is focused on a fixed position on the area of skin above the tissue to be studied. A frequency generating app is downloaded onto the i5 processor within the VOCT device. This app is capable of driving the speaker between 30 and $20,000 \mathrm{~Hz} \cdot{ }^{17-21}$ The speaker is placed near but not touching areas of the skin. During in vivo measurements, no sensation of the light or sound is felt impinging on the skin.

An OCT scanning image of the skin is obtained to ensure that the epidermis and dermis are normal. ${ }^{18-21}$ The spectral-domain optical coherence tomography (SD-OCT) system uses a fiber-coupled super luminescent diode light source with an $810 \mathrm{~nm}$ center wavelength and $100 \mathrm{~nm}$ bandwidth (full-width at half maximum). ${ }^{17-21}$ For measurement of the resonant frequency of a material, the VOCT system is operated in the $\mathrm{B}$ mode with the beam focused on one point with an area of about $14 \mu \mathrm{m}$ as previously indicated. ${ }^{17-21}$
The resonant frequency of each sample is determined by measuring the displacement of skin resulting from sinusoidal driving frequencies ranging from 30 to $200 \mathrm{~Hz}$, in steps of $10 \mathrm{~Hz}$. The peak frequency (the resonant frequency), $f_{n}$, is defined as the frequency at which the displacement is maximized as discussed previously. ${ }^{17-21}$ The moduli of skin and other tissues listed in Table 1 are calculated from measurements of $f_{n}$ using equation (1) for tissues. The material's modulus $(E)$, determined from these vibrational measurements, is related to the resonant frequency $f_{n}$ through equation (1) where $m, L$, and $A$, are the mass, length, and cross-sectional area of the sample, respectively as discussed previously. ${ }^{17-21}$

$$
E=m\left(2 \pi f_{n}\right)^{2}\left(\frac{L}{A}\right)
$$

Moduli determined using equation (1) were plotted against the corresponding moduli obtained from the tangent to the tensile stressstrain curves for all soft tissues tested as previously discussed ${ }^{17-21}$ and these data were used to develop equation (1a) for soft tissue and to calibrate the VOCT device.

$$
\begin{aligned}
& \text { Soft Tissue } \\
& \qquad E * d=0.0651 *\left(\mathrm{fn}^{2}\right)+233.16
\end{aligned}
$$

The skin thickness, $d$, is determined from the OCT scanning image. The weighted displacement versus frequency curve becomes a mechanical and vibrational spectrum generated by the components of the tissues that are vibrated. ${ }^{19-21}$ Weighted displacement is normalized by dividing by the displacement of the speaker in the absence of the sample to correct for the displacement as a function of frequency not due to the sample.

Table I Resonant frequency assignment for different components of skin. ${ }^{19}$ Note the exact resonant frequency of each skin component will depend on the skin thickness. However, the tissue stiffness(modulus) obtained from the resonant frequency and thickness is independent of the tissue thickness.

\begin{tabular}{lll}
\hline Component & $\begin{array}{l}\text { Resonant frequency } \\
(\mathbf{H z})\end{array}$ & Stiffness (MPa) \\
\hline Blood / Blood Vessels & 150 & 3.66 \\
Cells & $50-70$ & 1.11 \\
Cartilage & 290 & 16.2 \\
Dermal Collagen & $90-120$ & 2.15 \\
& & 10.84 \\
Fibrotic Collagen & $200-220$ & 15.9 \\
\hline
\end{tabular}

\section{Results}

In this study, we present the VOCT results of skin treated with a topical agent (CE Ferulic) and micro-needling. Needling creates small holes in the epidermis and dermis leading to a wound healing response as apposed to topical application which does not cause bleeding or create a hole in the skin (see Figures 1 through 3 ). The weighted displacement versus frequency data and OCT images 
obtained initially and at 1 and 7 months post-treatment with the topical preparation are shown in Figure 1. The OCT images on the right of Figure 1 illustrate that the skin is composed of an epidermis (yellow and pink colors) and papillary dermis (blue color). The epidermal stratum corneum surface (yellow) appears to reflect less light as the treatment time increases. The pixel intensity from these images is plotted versus depth as shown in Figure 2. Note the pixel intensity of the surface layer of stratum corneum decreases (yellow color) with treatment time suggesting that this layer is thinner as a result of $\mathrm{CE}$ Ferulic treatment. On the left hand side of Figure 1 are shown plots of weighted displacement versus resonant frequency for the different treatment times. Each peak represents the vibration of a tissue component listed in Table1. Note the exact resonant frequency of each component depends on the skin thickness whereas the component modulus is independent of thickness. In the zero time control there is a small cellular peak at about $70 \mathrm{~Hz}$ and large dermal collagen peak at about $130 \mathrm{~Hz}$. A reorganized collagen peak, typical of fibrous tissue (scar tissue) deposition, is not seen initially (Figure 1). At the 1 month and longer treatment time periods, the cellular peak and the fibrous collagen peaks appear to grow at the expense of the dermal collagen peak. Table 2 lists values of the ratio of the cellular to dermal collagen peaks indicating that the cellular peak is normally a small fraction of the dermal collagen peak in the absence of blood vessels. Table 3 shows that the ratio of the cellular to dermal collagen peak as well as the ratio of the fibrous collagen peak to dermal collagen peak increases with increasing treatment time indicating that new fibrotic collagen is being deposited.
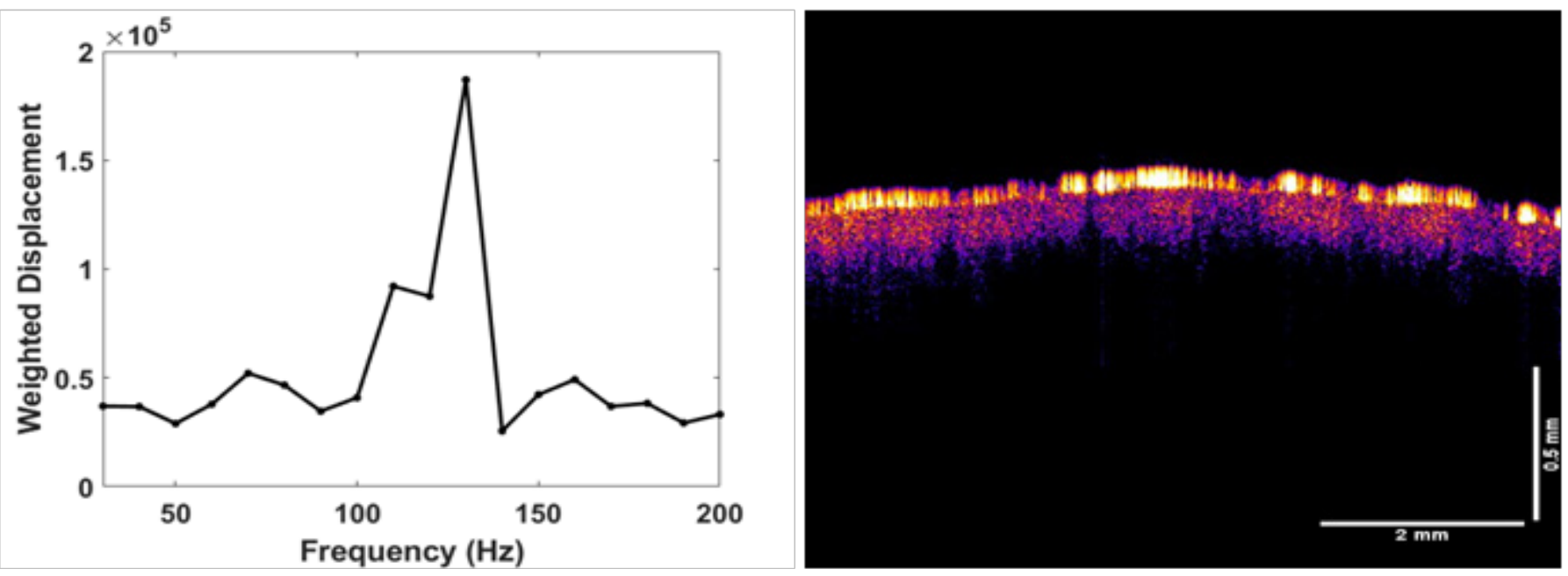

Figure la
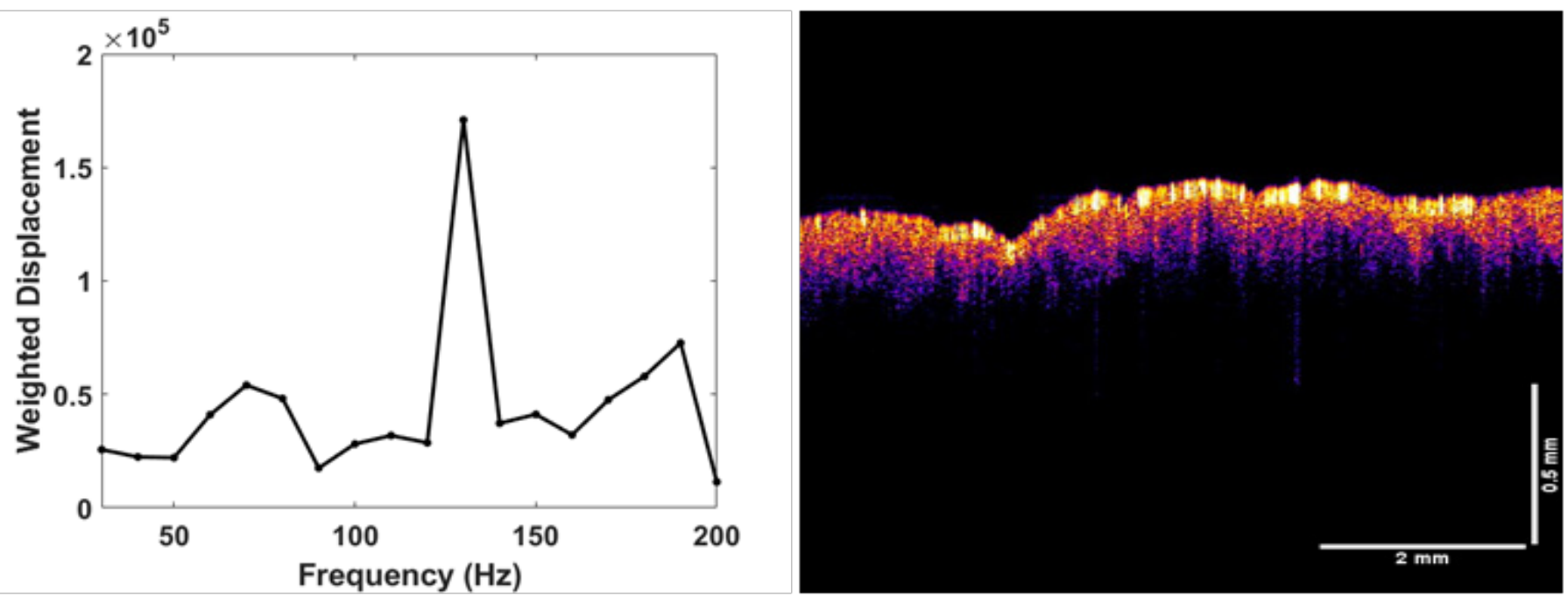

Figure Ib 

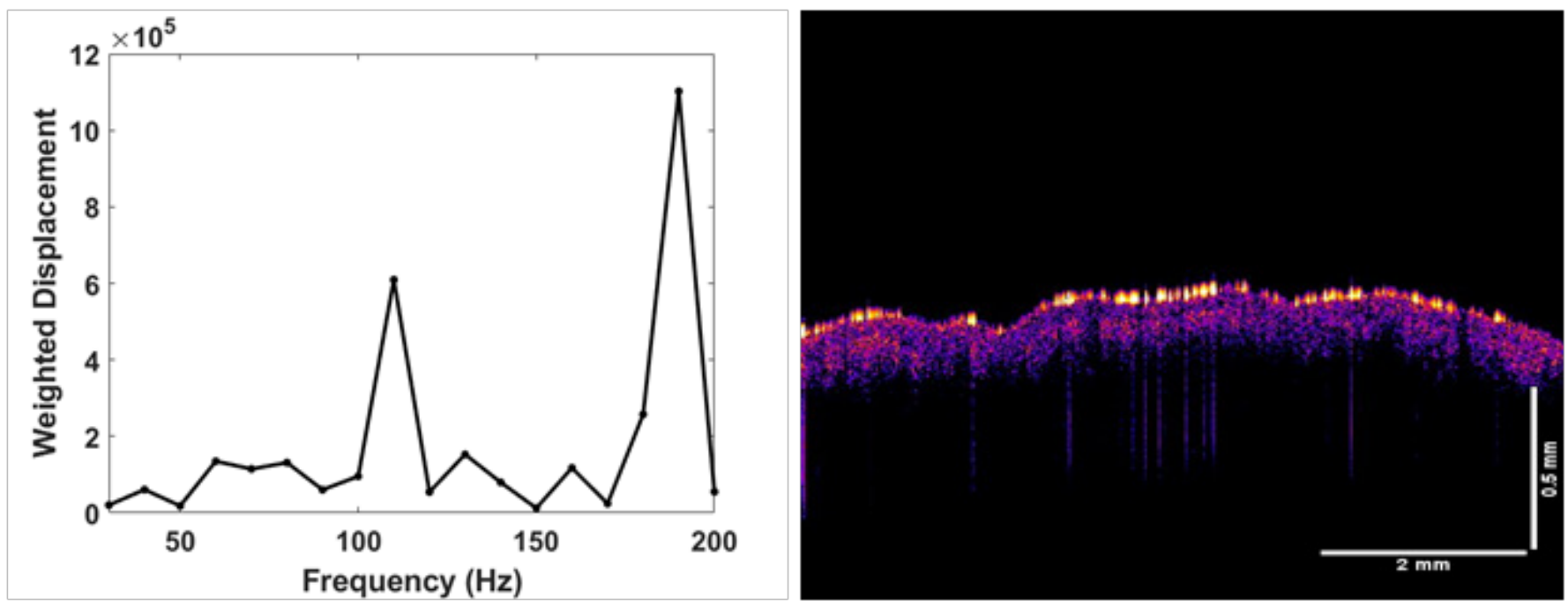

Figure Ic

Figure I Weighted displacement versus frequency (left) and OCT image of forehead skin (right) treated with CE Ferulic daily: (a) zero-time control before treatment; (b) treatment time I month; and (c) treatment time 7 months. Note the reflection of the stratum corneum (yellow) decreases with increasing time indicating that this layer is changing with time. The peaks identified include the cellular peak $(50-70 \mathrm{~Hz})$, dermal collagen $(90-\mathrm{I} 20 \mathrm{~Hz})$ and fibrotic collagen $(200 \mathrm{~Hz})$. Note the increase in cellular $(50-70 \mathrm{~Hz})$ and fibrotic peaks $(200 \mathrm{~Hz})$ with increasing treatment times. CE Ferulic treatment appears to increase the epidermal cellular content and fibrous collagen deposition in the skin.

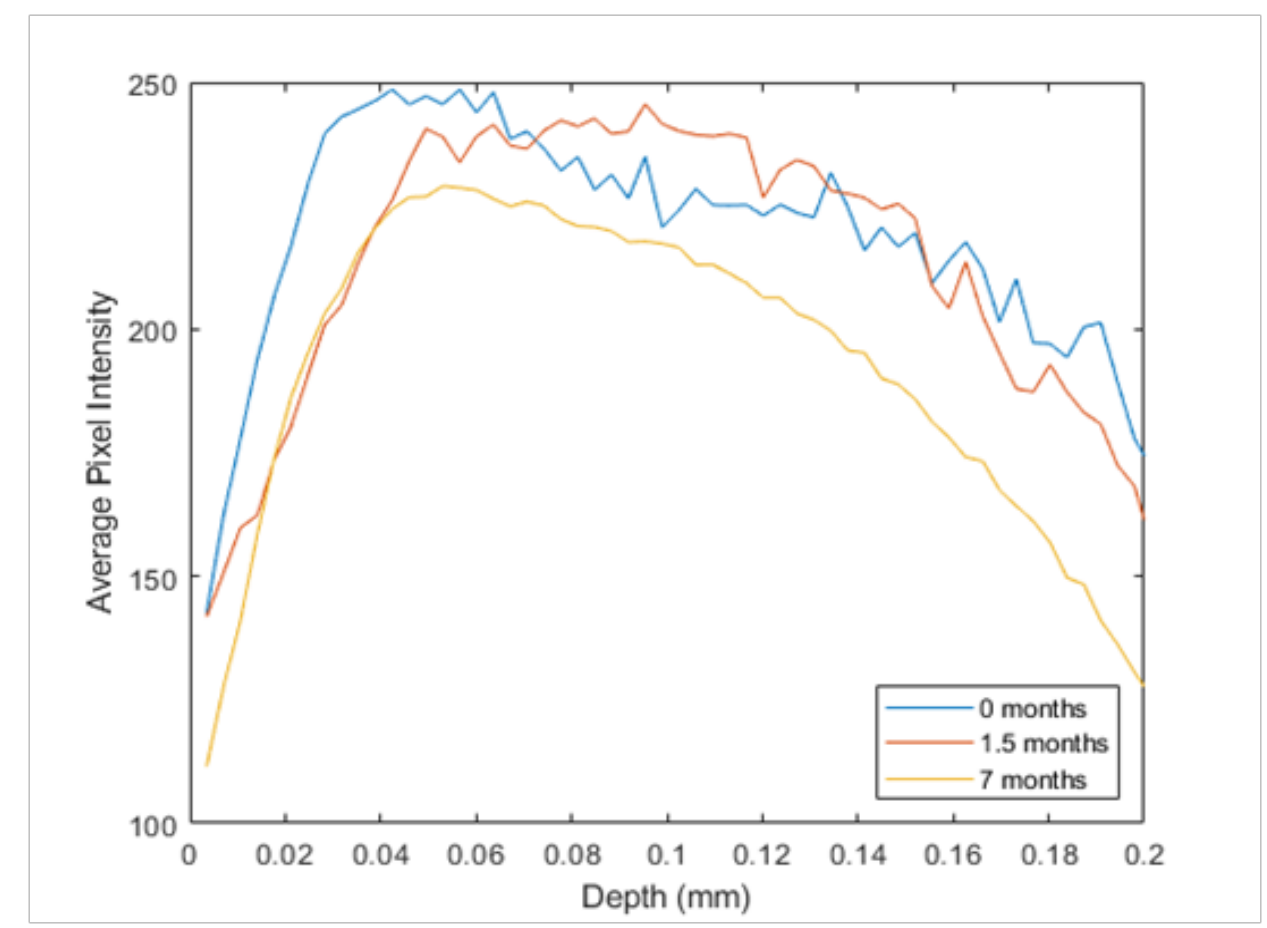

Figure 2 Pixel intensity versus depth of skin treated topically with CE Ferulic. Note the pixel intensity appears to decrease with treatment time due to changes in the stratum corneum. Topical treatment also leads to new epidermal cells and new fibrotic collagen (Figure I) (Table 3).

Figure 3 shows a comparison of the OCT images (right) for different treatment times for the skin that is micro-needled using a roller. The OCT images just before and after treatment illustrate that the needle holes are difficult to observe after the needle is removed. Only traces of clotted blood (circle) and a needle hole (arrow) define where the needle penetrated the skin. Needling did appear to generate a peak at $150 \mathrm{~Hz}$ indicative of blood and blood vessels (Table 2) that appeared to remain for periods up to two weeks. Figure 4 is a plot of pixel intensity versus depth for the micro-needled skin. Note the pixel intensity appears to increase initially due to inflammation; however, it does appear to go back to normal with increased time once inflammation is resolved. Eventually, using the topical treatment, the 
stratum corneum appears to thin. This does not seem to be the case after needling. After needling the thickness of the stratum corneum is similar to the unneedled skin at time zero. The OCT images show that the reflectivity of the stratum corneum appears to decrease with increased time after treatment. After reviewing the data in Table 3,

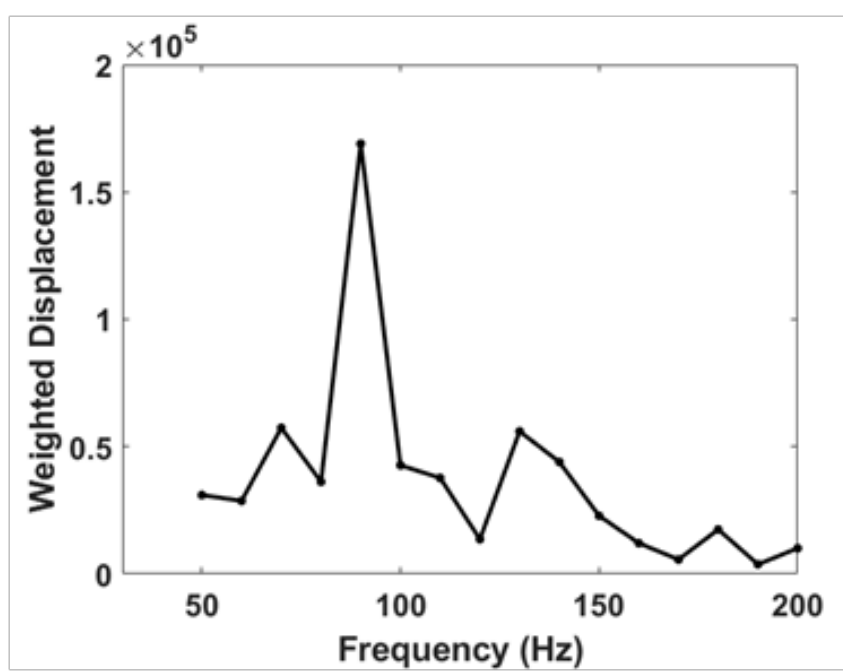

needling as does topical treatment, increases the ratio of the cellular to dermal collagen peaks and the ratio of fibrotic collagen to dermal collagen peaks. Therefore, both of these techniques appear to rejuvenate skin by inducing a wound healing response that causes cell proliferation and collagen synthesis.

Figure 3a
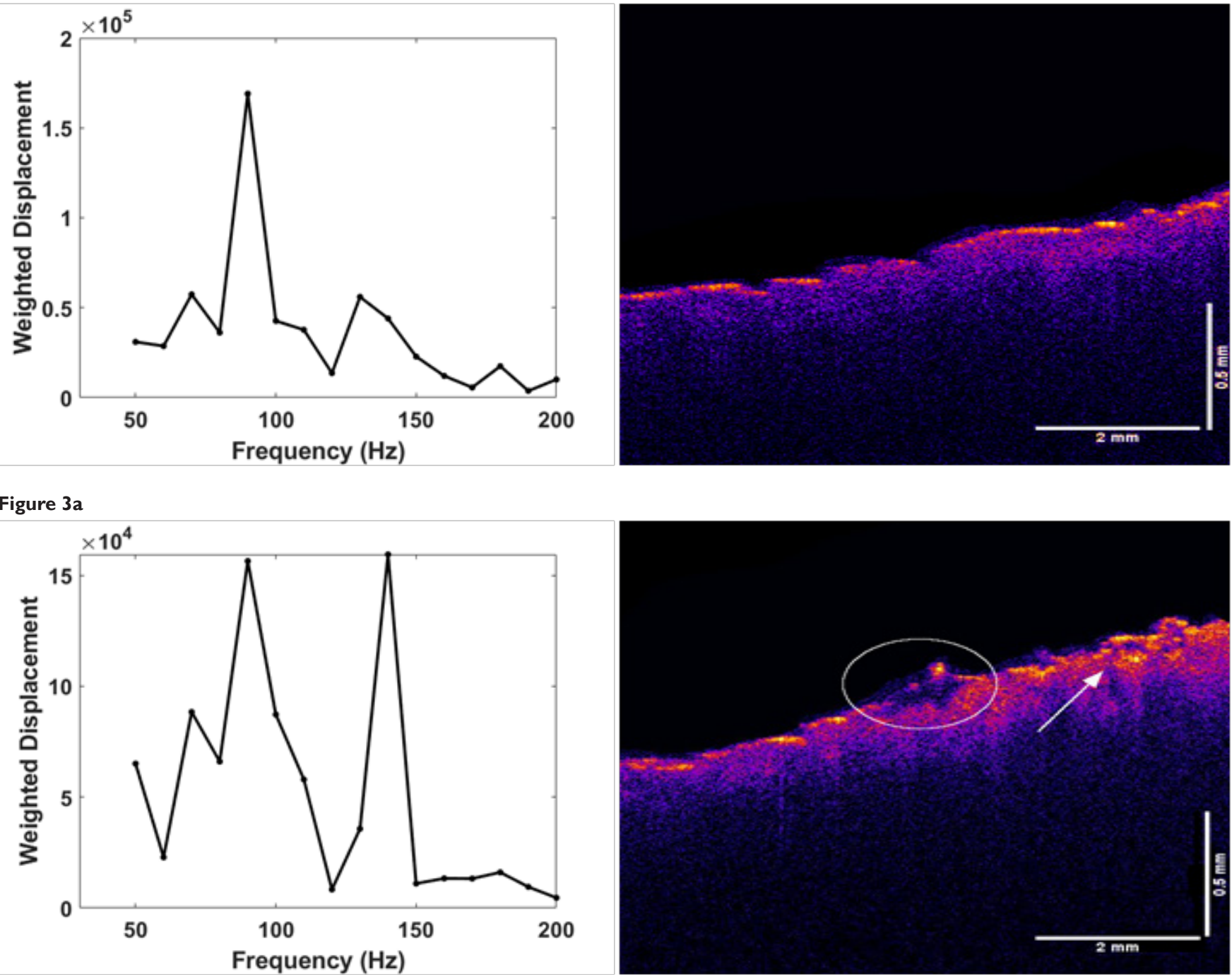

Figure 3b
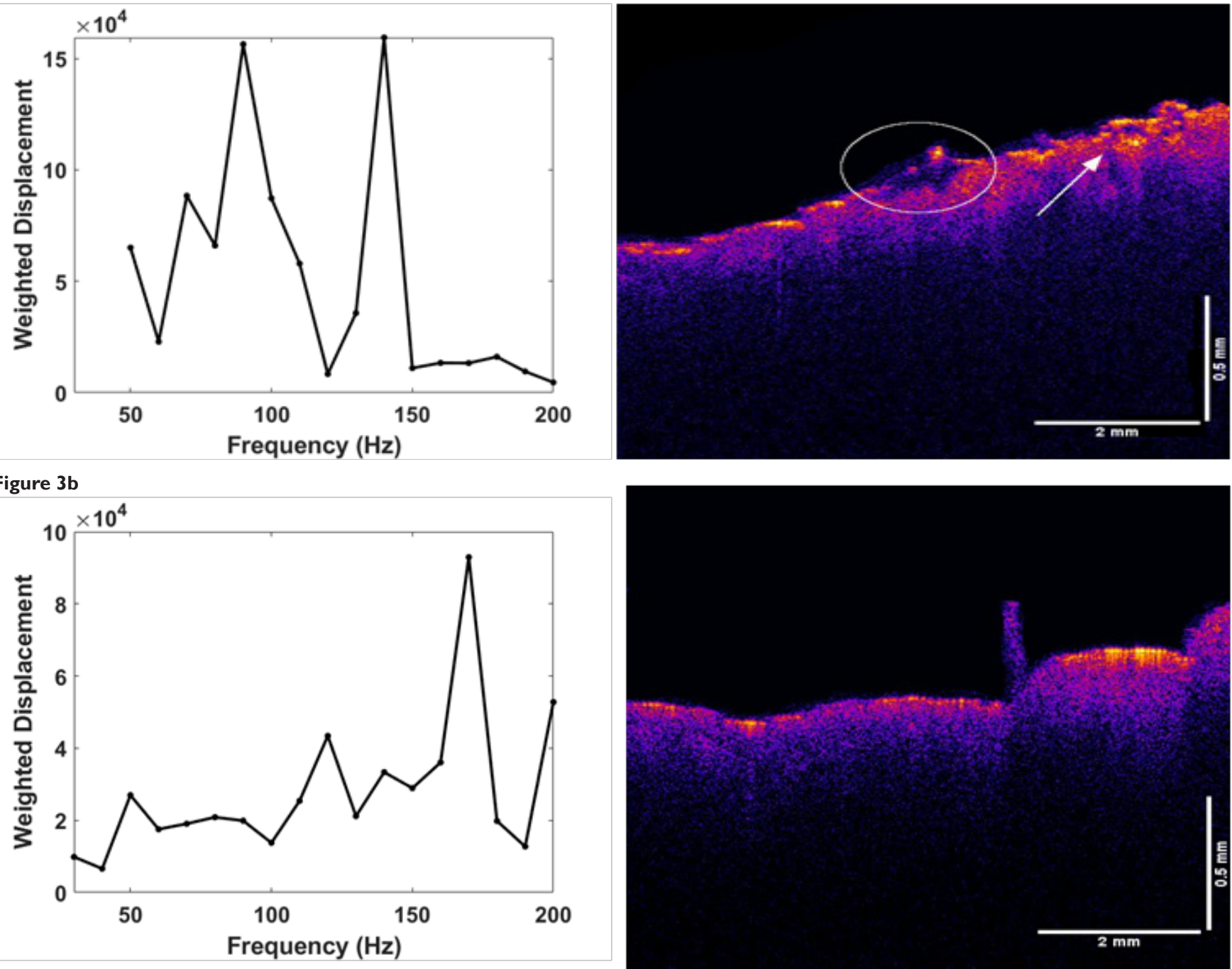

Citation: Silver FH, Kelkar N.Virtual biopsies and vibrational-mechanical analysis of skin rejuvenation: comparison of results obtained with micro-needling and topical treatments.J Dermat Cosmetol. 2020;4(3):50-57. DOI: I0.15406/jdc.2020.04.00I5 I 
Figure 3c

Figure 3 Weighted displacement versus frequency (left) and OCT image of forehead skin (right) rollered with a micro-needle observed at different post-treatment time intervals after needling: (a) zero time control before treatment; (b) immediately after treatment; and (c) 4 weeks after treatment. Note the blood (circle) and needle hole (arrow) in the image immediately after treatment (b). The blood clot and needle hole disappear after about one week. New epidermal cells and new fibrous collagen form faster after needle roller treatment compared to topical treatment with CE Ferulic.

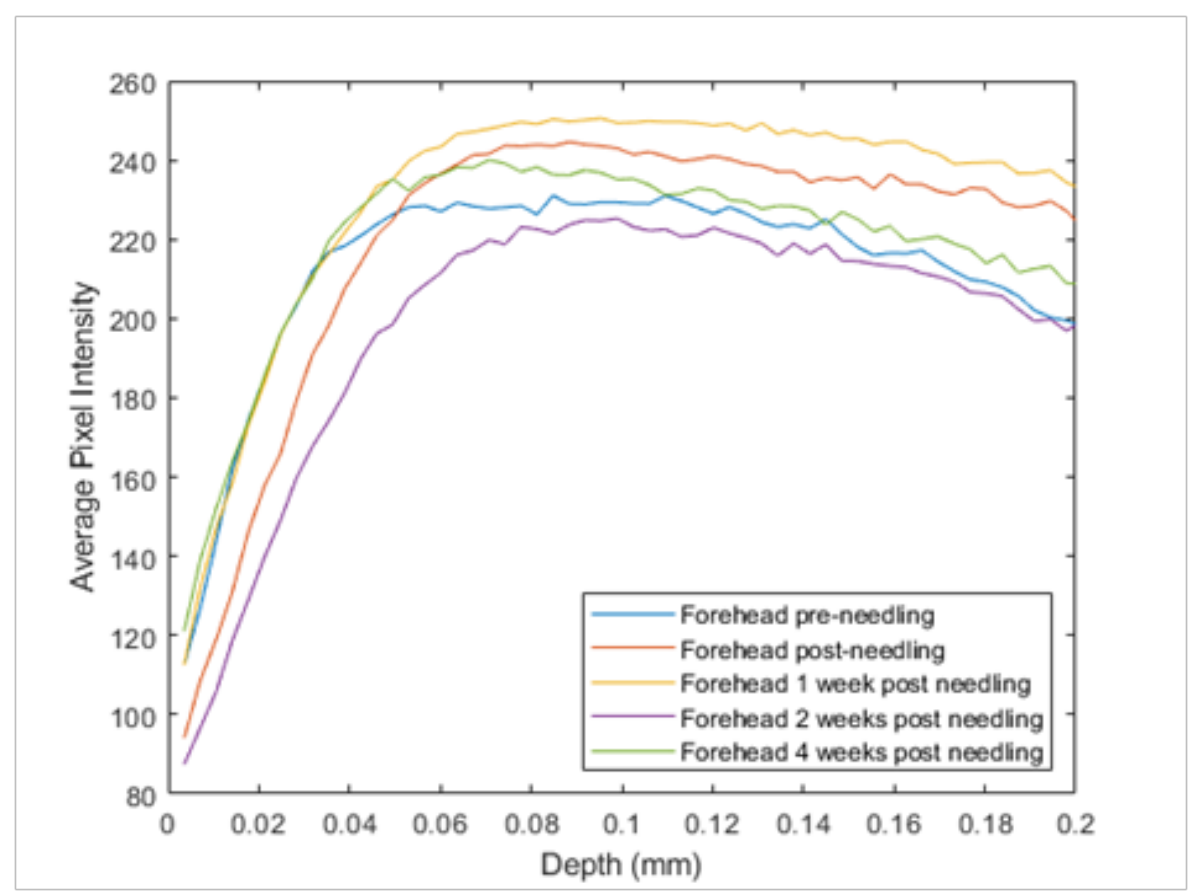

Figure 4 Pixel intensity versus skin depth treated topically by micro-needle rolling. Note the pixel intensity appears to decrease with treatment time due to resolution of inflammation that occurs immediately after needling. Needling produces a rapid wound healing response that leads to new epidermal cells and new fibrous collagen that result from creating a wound in the epidermis and dermis.

Table 2 Resonant Frequency and Ratio of Cellular to Collagen Resonant Frequency Peak Height for Different Locations of Human Skin at various locations. ${ }^{17-21} \mathrm{~Hz}$ equals Hertz; ratio equals cell resonant frequency peak height at $50-70 \mathrm{~Hz} /$ collagen resonant frequency peak height

\begin{tabular}{lll}
\hline Skin location & Resonant frequency $(\mathbf{H z})$ & Ratio \\
\hline Wrist & $70,110,155$ & 0.189 \\
Forehead & 75,134 & 0.061 \\
Cheek & 80,140 & 0.008 \\
Palrn & $60,150,170$ & 0.04 \\
\hline
\end{tabular}

\section{Discussion}

A great deal of research has been directed at finding new methods to improve the diffusion of hydrophilic components through the stratum corneum-granulosum junction into the dermis. This has led to development of techniques to promote formation of new collagen and repair of the epidermis. However, there are no quantitative techniques available to image and measure the changes that occur to the major skin components during these procedures. As a result of the lack of techniques available to evaluate skin treatments, optimizing skin rejuvenation is a trial and error process. Skin rejuvenation evaluation would be facilitated by development of a technique that was able to image and quantitatively measure any changes that occur to the epidermis and dermis.

The results of this study show that VOCT is a technique that can be used to measure changes in epidermal and collagen components in the dermis. This technique has been used to characterize thermal and chemical burns, skin cancers, implant materials, biofilms, and both soft and hard tissues. ${ }^{17-21}$ VOCT provides images and mechanical measurements of internal tissues as deep as 1.0 to $2.0 \mathrm{~mm}$ in real time. ${ }^{18-21}$ VOCT in combination with high frequency ultrasound can be used to collect images and mechanical data on tissues as deep as $8.0 \mathrm{~cm} \cdot{ }^{19}$

Our results suggest that both topical skin treatment and microneedle rolling of the forehead appear to produce changes in both the epidermis and dermis. The changes occur by either diffusion through the stratum corneum-stratum granulosum barrier or by penetrating through it by creating holes. Both treatments increase the ratio of the cellular peak to the dermal collagen peak and the ratio of the fibrotic collagen peak to the normal dermal collagen peak observed (Table 3). However, micro-needling does it more rapidly but also involves some pain associated with the trauma as the needles are inserted through the epidermis into the dermis. In normal skin, in the absence of blood vessels, the ratio of cellular to dermal collagen resonant frequency peak heights is very small (Table 2). However, after both topical and needle treatment this ratio increases (Table 3) suggesting 
that the number of cells present in the treated area increases as well as the vasculature after needling. The observation that both of these treatments increase the number of cells in the epidermis below the stratum corneum suggests that both processes create a wound healing response. This is supported by the observation that the ratios of the fibrotic collagen peak to dermal collagen peak increases with time (Table 3). This result suggests that the new collagen formed is composed of fibrotic collagenous tissue and not normal dermal tissue. Normal dermal collagen consists of thin collagen fibrils that form fibers in a biaxial network within the plane of the skin. In fibrous scar tissue, collagen forms an aligned network similar to that seen in tendon. ${ }^{22}$ The consequence of depositing fibrotic collagen versus dermal collagen via topical application or needling is that ultimately too much fibrous tissue will cause stiffening of the skin and limit the ability of skin to deform when subject to mechanical loading. ${ }^{22-24}$ Fibrotic scar tissue tears easily compared to normal skin, since normal skin can dissipate more energy by stretching when mechanically deformed. ${ }^{22-24}$ The fact that scar tissue stretches less easily than normal dermal collagen is well-known to boxers since facial scars tear more easily than normal skin when hit during a fight.

Table 3 Resonant frequency peak height ratios of cell $(50$ to $70 \mathrm{~Hz})$ to dermal collagen $(90$ to $\mathrm{I} 20 \mathrm{~Hz})$ and for reorganized (fibrotic) collagen $(200$ to $220 \mathrm{~Hz})$ to dermal collagen $(90$ to $120 \mathrm{~Hz})$ for CE Ferulic topical treatment versus micro-needle rolling of forehead skin versus time after treatment.

\begin{tabular}{llll}
\hline Treatment & $\begin{array}{l}\text { Time } \\
\text { (months) }\end{array}$ & $\begin{array}{l}\text { cell/ } \\
\text { dermal } \\
\text { coll }\end{array}$ & $\begin{array}{l}\text { Reorg coll/ } \\
\text { dermal coll }\end{array}$ \\
\hline Topical CE Ferulic & 0 & 0.0667 & 0.0625 \\
& 1.5 & 0.118 & 0.034 \\
& 6.5 & 0.417 & 0.66 \\
Treatment & 7 & 0.182 & 1.82 \\
& Time & $\begin{array}{l}\text { cell/ } \\
\text { dermal } \\
\text { coll }\end{array}$ & $\begin{array}{l}\text { Reorg coll/ } \\
\text { dermal coll }\end{array}$ \\
& Meeks) & 0.231 & 0.0769 \\
& 0 Pre & 0.33 & 0.0667 \\
& 0 Post & 0.33 & 0.0833 \\
& I & 0.33 & 1.1 \\
& 2 & 0.667 & 1.36 \\
\hline
\end{tabular}

It is now possible using VOCT to generate images and physical data to quantitatively assess cell proliferation and collagen deposition at different time periods. This will lead to better evaluation of the outcomes and optimization of skin treatments. The efficacy of outcomes and treatment methods using lasers, radiofrequency and ultrasound can be tailored to each patient based on VOCT data. It is well known that there is a patient-to-patient variation in the response to injury and wound healing that depends on the underlying biological responses. Using VOCT, the physician or aesthetician can evaluate each client's response to a treatment and tailor the outcome based on the subject's internal skin response.
In addition to optimizing skin care protocols, VOCT will be useful in developing techniques to find methods to revitalize skin by depositing dermal collagen as opposed to fibrotic collagen. This is important in areas of skin such as contour defects and areas with rapid changes in the radius of curvature where increased collagen stiffness will lead to poor functional outcomes. Examples of these cases include scarring over joint surfaces and contractures that occur in the neck of burn patients.

\section{Conclusions}

VOCT, a non-invasive and non-destructive technique, has been used to quantitatively evaluate the outcomes of cell proliferation and fibrous tissue deposition as a result of a skin topical treatment and after micro-needle rolling. Both methods appear to quantitatively increase the ratio of epidermal cells to dermal collagen and the ratio of fibrotic collagen to dermal collagen. These results indicate that chemical or mechanical treatment can lead to cell proliferation and new collagen deposition but that the collagen deposited is more like fibrous scar tissue than collagen in papillary and reticular dermis. While microrolling creates new fibrotic collagen more rapidly, it is also associated with more trauma and pain.

The new collagen that is deposited appears similar to that seen in wound healing and has a higher resonant frequency and modulus compared to normal dermal collagen. Using VOCT it will be possible to develop skin rejuvenation techniques that promote the deposition of dermal collagen and limit fibrosis. In addition, use of VOCT after aesthetic procedures will allow optimization of skin rejuvenation that is patient and treatment specific.

\section{Conflits of interest}

The author declares that there is no conflicts of interests.

\section{Acknowledgments}

None.

\section{Funding}

None.

\section{References}

1. Ramose Silva M, Celem LR, Ramose Silva AP. Anti-aging cosmetics: Facts and controversies. Clin Dermatol. 2013;31(6):750-758.

2. Beasley KL, Weiss MA, Weiss RA. Hyaluronic Acid Fillers: A Comprehensive Review. Facial Plas Surg. 2009;25(2):86-94.

3. Baumann L, Saghari S, Weisberg E. Retinoids: In: Cosmetic Dermatology, Principles and Practice. chapter 30, McGraw Medical, USA. 2000;256-262.

4. Wei JCJ, Haridass IN, Crichton ML, et al. Space- and timeresolved investigation on diffusion kinetics of human skin following macromolecule delivery by microneedle arrays. Scientific Reports. 2018;8:17759.

5. Tsai TC. Basil M. Hantash BM. Cosmeceutical Agents:AComprehensive Review of the Literature. Clinical Medicine: Dermatology. 2008;1:120 .

6. Brown IA. Scanning electron microscopy of human dermal fibrous tissue. J Anat. 1972;113(2):159-168. 
7. Smith LT, Holbrook KA, Byers PH. Structure of the dermal matrix during development and in the adult. J Invest Dermatol. 1982;79:93104.

8. Lavker RM, Zheng P, Dong G. Aged skin: a study by light, transmission electron, and scanning microscopy. J Invest Dermatol. 1987;88:44s-51s.

9. Cotta Pereira G, Rodrigo G, Bittencourt-Sampaio S. Oxytalin, elaunin, and elastic fibers in the human skin. J Invest Dermatol. 1976;66(3):143148.

10. Vitellaro-Zuccarello L, Cappelletti S, Poozo Rossi VD, et al Stereological analysis of collagen and elastic fibers in the normal human dermis: variability with age, sex, and body region. Anat Rec. 1994;8(2):153-162.

11. Menon GK, Cleary GW, Lane ME. The structure and function of the stratum corneum. Int J Pharm. 2012;435(1):3-9.

12. Prausnitz MR, Langer R.Transdermal drug delivery. Nat Biotechnol. 2008; 26(11):1261-1268.

13. Kalluri H, Banga AK. Transdermal Delivery of Proteins. AAPS Pharm Sci Tech. 2011;12(1):431-441.

14. Pastore MN. Yogeshvar N. Kalia YN. et al. Transdermal patches:history, developmentand pharmacology. British Journal of Pharmacology. 2015;172(9):2179-2209.

15. Seah BC-Q, Boon MT. Recent advances in ultrasound-based transdermal drug delivery. Int J Nanomedicine. 2018;13:7749-7763.

16. Yang G, Zhang Y, Gu Z. Punching and Electroporation for Enhanced Transdermal Drug Delivery. Theranostics. 2018;8(13):3688-3690.

17. Silver FH, Shah RG, Richard M. Comparative "virtual biopsies" of normal skin and skin lesions using vibrational optical coherence tomography. Skin Res Tech. 2019;25:743-749.
18. Silver FH, Kelkar N, Deshmukh T. et al. Virtual Biopsies" of Normal Skin and Thermaland Chemical Burn Wounds. Adv Skin Wound Care. 2020;33(6);307-312.

19. Silver FH. Kelkar N. Desmukh T. et al. Mechano-Vibrational Spectroscopy of Tissues and Materials Using Vibrational Optical Coherence Tomography: A New Non-Invasive and Non-Destructive Technique. Recent Progress in Materials. 2020;2(2).

20. Silver FH, Shah RG, Richard M, et al. Comparison of the virtual biopsies of a nodular basal cell carcinoma and an actinic keratosis: Morphological, cellular and collagen analyses. Adv Tissue Eng and Regen Med. 2019; 5:61-66.

21. Silver FH, Shah RG. Richard M Benedetto D. Use of vibrational optical coherence tomography to image and characterize a squamous cell carcinoma. J Derm Res Ther:2019; 5: 067.

22. Doillon CJ, Dunn MG, Bender E, et al. Collagen Fiber Formation In Vivo: Development of Wound Strength and Toughness. Collagen and Related Research. 985;5(6):481-492.

23. Dunn MG Silver FH. Viscoelastic behavior of human connective tissues: relative contribution of viscous and elastic components. Connect Tissue Res. 1983;12(1):59-70.

24. Dunn MG. Silver FH. Swann DA. Mechanical Analysis of Hypertrophic Scar Tissue: Structural Basis of Apparent Increased Rigidity. J Invest Dermatol. 1985;84(1):9-13. 